\title{
DEPOSITO VOTIVO EN UN YACIMIENTO DE LA EDAD DEL BRONCE EN EL VALLE DEL MANZANARES (PERALES DEL RIO, GETAFE, MADRID)
}

\author{
M. ${ }^{\text {a }}$ CONCEPCIÓN BLASCO \\ M. ${ }^{a}$ LUZ SÁNCHEZ-CAPILLA \\ PATRICIA CAPRILE \\ JUANA CALle
}

La ausencia de datos con la que, hasta hace poco, contábamos para la Edad del Bronce en el área oriental de la Meseta, empieza ahora a ser contrarrestada por el constante descubrimiento de yacimientos y hallazgos sueltos enmarcables en esta etapa de la Prehistoria reciente aunque, desgraciadamente, todos estos nuevos datos adolecen, en la mayoría de los casos, de un estudio detenido debido casi siempre al estado de arrasamiento en el que llegan hasta nosotros dichos yacimientos y a las escasas excavaciones que en ellos se han realizado. Por estas causas, el conocimiento que tenemos de la forma de vida, las costumbres y creencias de las gentes asentadas en esta región oriental de la Meseta durante la Edad del Bronce, sigue siendo muy fragmentario y, ni siquiera es posible, todavía hoy, contar con una secuencia cronológico-cultural suficientemente contrastada que permita ubicar, con un mínimo de garantía, los sucesivos hallazgos que se van produciendo.

$\mathrm{El}$ estado actual de conocimientos nos permite apuntar que, al menos en las tierras de la cuenca del complejo Henares-Jarama, donde se encuadran buena parte de las actuales provincias de Madrid y Guadalajara, existen durante el Bronce Medio y Final, al menos, dos complejos o facies culturales cuya diacronía o sincronía no está plenamente aclarada (1). El elemento diferenciador de estos dos grupos es la cerámica ya que en el primero de ellos dominan los recipientes lisos con carenas medias, medias-altas o bajas bien marcadas jalonadas, a veces con pezones realizados con aplicaciones plásticas, mientras que en el segundo complejo encontramos, con bastante frecuencia, recipientes ornamentados con técnicas muy variadas, muchas veces combinadas en el mismo ejemplar; entre ellas cabe citar incisiones, impresiones, boquique, excisiones, etc. Esta diferencia de los ajuares cerámicos no parece reflejar, en cambio, un comportamiento cultural muy distinto cosa lógica, por otra parte, si pensamos que no existe una situación económica y social demasiado alejada.

Dentro del esquema tradicional de la Prehistoria peninsular, los grupos con cerámicas carenadas lisas corresponderían a un Bronce Clásico, identificado como

(1) Blasco Bosqued, M. C.: “El Bronce Medio y Final» en 130 años de arqueología madrileña, Madrid, 1987, pp. 83-101. 
Bronce Medio, mientras que aquéllos que ornamentan sus vasijas más elaboradas con diseños geométricos realizados con distintas técnicas de incrustración pertenecen, en su mayor parte, a un Bronce Tardío-Final. Estos últimos han proporcionado conjuntos con ciertas matizaciones diferentes que permiten atisbar una evolución desde un primer momento, con escasa presencia de ejemplares ornamentados y desarrollo de temas decorativos simples en los que falta la excisión y el boquique hasta las etapas más evolucionadas en las que se produce un gusto por la profusión ornamental y las composiciones barroquizantes en las que intervienen distintas técnicas dentro de un mismo recipiente y que, a veces, son soporte que permiten la inscrustración de sustancias colorantes cuyas pigmentaciones contrastan con el tono oscuro de las paredes de las vasijas.

Estas facies culturales desarrolladas durante el Bronce Tardío y Final de la Meseta, conocida en la bibliografía científica como Horizonte Cogotas I, entroncaría con el Bronce Clásico a través de esa primera subfase con cerámicas de decoraciones sencillas, que suponen bajos porcentajes en los conjuntos generales y a la que hemos denominado etapa Protocogotas (2) la cual parece estar bien representada en el área que nos ocupa y en la que se encuadran plenamente los restos arqueológicos que estudiamos en este trabajo.

Dichos restos proceden de la finca denominada La Torrecilla, limítrofe con «Perales del Ríon, zona que desde hace tiempo es pródiga en restos arqueológicos. El yacimiento que nos ocupa está situado a la altura del kilómetro 8,900, izquierda, de la carretera a San Martín de la Vega; se localiza en la Hoja 582, correspondiente a Getafe, del mapa 1:50.000 del I.G.N. en la intersección de las coordenadas 3 38' 10"' Longitud W, 40 19' 40" Latitud Norte.

En esta finca se han cartografiado, entre otros, los siguientes restos arqueológicos: necrópolis visigoda excavada y publicada por M. C. Priego y S. Quero (3); necrópolis romana de incineración excavada y publicada por R. Lucas, C. Blasco y M. A. Alonso (4); villa romana, todavía en curso de excavación por estas mismas autoras (5); posible "campo de urnas" publicado por S. Quero y M. C. Priego (6); yacimiento de la Edad del Bronce con restos tardorromanos superpuestos, excavado por M. L. Cerdeño, A. Méndez y otros (7), a lo que ahora se suma este nuevo yacimiento.

(2) Esta fase también ha sido identificada en algunos yacimientos en la Meseta Norte, donde se le ha denominado facies Cogeces; vid. Delibes, G. y Fernández Manzano, J.: “El Castro protohistórico de "La Plaza", en Cogeces del Monte (Valladolid). Reflexiones sobre el origen de la fase Cogotas I", B.S.A.A., tomo XLVII, Valladolid, 1981, págs. 51-69.

(3) Priego, M. C. y Quero, S.: "Noticias sobre la necrópolis visigoda de La Torrecilla (La Aldehuela, Madrid)", en XIV Congreso Nacional de Arqueologia, Zaragoza, 1977, págs. 1.261-1.264.

(4) Lucas, R. et alii: «Necrópolis romana de La Torrecilla (Getafe, Madrid)», en Noticiario Arqueológico Hispanico, n..$^{\circ}$, Madrid, 1982, págs. 213-246.

(5) Lucas, R.; Blasco, C. y Alonso, M. A.: "El hábitat romano de La Torrecilla (Getafe, Madrid)", en Noticiario Arqueológico Hispánico, n. ${ }^{\circ}$ 12, Madrid, 1981, págs. 375-390.

(6) Priego, M. C. y Quero, S.: «Una obra maestra de la orfebrería prehistórica madrileña: el brazalete 'de oro de La Torrecilla (Getafe, Madrid)", en Villa de Madrid, año XVI, 1978-2, 59, Madrid, 1978, págs. 17-23.

(7) Cerdeño, M. L. et alii: «El yacimiento de la Edad del Bronce de La Torrecilla (Getafe, Madrid)», en Noticiario Arqueológico Hispánico, n. ${ }^{\circ}$ 9, Madrid, 1980, págs. 151-242. 
Recientemente se han localizado, también en las proximidades de este paraje, un asentamiento campaniforme, un posible taller de sílex del Paleolítico inferior y otro yacimiento más del Horizonte Cogotas I.

La localización del hallazgo que nos ocupa se produjo al detectarse la existencia de dos recipientes cerámicos colocados, con la boca hacia abajo, en el corte de un arenero, abierto hace varios años y abandonado hace ya bastante tiempo. Esta localización es consecuencia de las prospecciones llevadas a cabo en la zona para identificar todos los restos existentes en los cortes realizados por las máquinas que trabajan en la extracción de áridos.

En un primer momento se tuvo la sensación de que los recipientes estaban aislados, sin contexto alguno, pero una vez limpio el corte del arenero, se comprobó que se encontraban dentro de una mancha oscura, correspondiente a un típico "fondo". Ante esta constatación, se decidió excavar la bolsada con el fin de conocer el contexto en que aparecieron los dos recipientes completos, a pesar de la dificultad que entrañaba el trabajo, debido a la altura del corte del arenero y a la cantidad de tierra acumulada por las máquinas sobre la superficie del fondo. Este tipo de fondos o bolsadas oscuras, directamente excavadas en el subsuelo forman, habitualmente, agrupaciones más o menos extensas y son características de la mayoría de los yacimientos de la Prehistoria reciente de esta región.

La excavación se realizó por niveles artificiales de 20 centímetros. La primera acumulación importante de restos se produjo a unos 40-45 centímetros de la superficie; allí se localizaron diversos restos óseos de bos (pertenecientes, al menos, a dos individuos), y de ovicápridos así como un fémur de perro. $\mathrm{Al}$ menos parte de un ovicáprido ( 3 vértebras lumbares y el sacro) estaban en conexión anatómica lo que parecía indicar que no se trata de restos de consumo. En el mismo nivel se obtuvieron también cuatro pequeños fragmentos de cerámica, decorado el primero con incisión y puntillado (fig. 1-8), el segundo con ungulaciones (fig. 1-3; 6; 7); el tercero, correspondiente a un fragmento de boca, presenta un cordón en el labio y el cuarto un mamelón; estos elementos plásticos son los que, precisamente, han sido objeto de decoración impresa. A todo ello hay que añadir la existencia de una veintena más de pequeños fragmentos cerámicos poco significativos así como algunos cantos rodados y restos de tierra endurecida.

Bajo esta capa pródiga en hallazgos que se encontraba en la parte inferior del cuello de la bolsada, se localizaban los dos recipientes completos (figs. 1-1 y 2) colocados boca abajo y uno sobre otro. El más pequeño de ellos (fig. 1-2), que ocupaba la parte más baja, tenía en su interior pequeños fragmentos óseos de los que sólo ha podido ser identificada una costilla de bos. Ambos presentan un perfil simple, ligeramente en "S", con la boca indicada y base plana.

En la parte más baja de la bolsada, junto a la base, se obtuvieron varios fragmentos más de cerámica de los cuales dos están decorados (figs. 1-4 y 5), uno de ellos con una espiguilla impresa y el otro con una somera incisión que crea un motivo triangular.

El relleno geológico que colmataba la bolsada presentaba un color gris, bastante homogéneo en el que no era apreciable ningún estrato. Dicha bolsada presenta- 


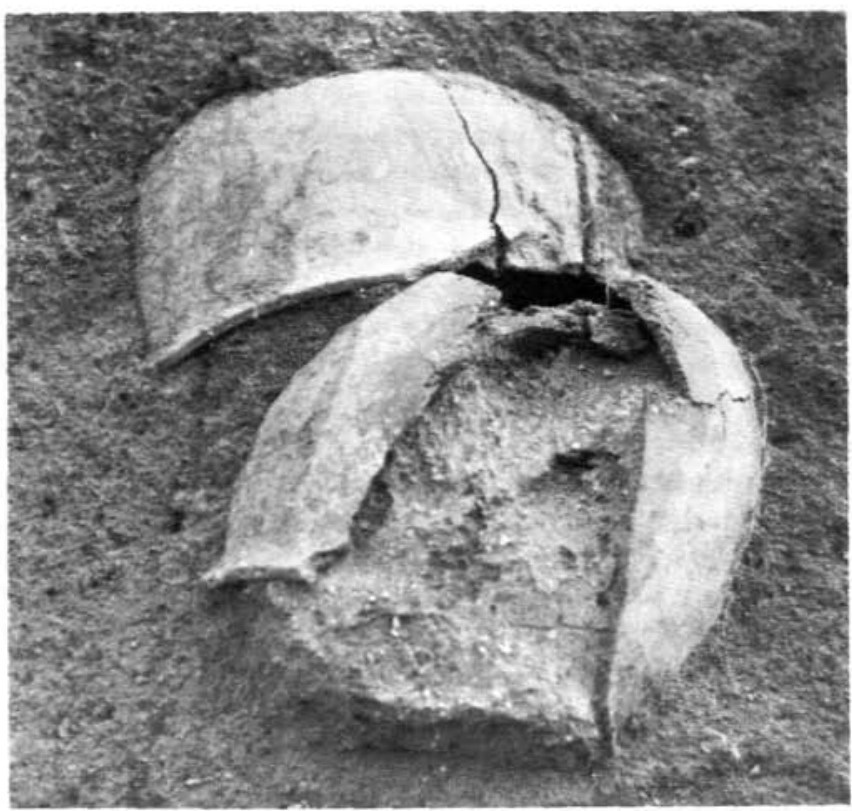

LÁmINA 1-1--Recipientes completos encontrados en el fondo número 1, en el momento de su descubrimiento en el corte del arenero abandonado.

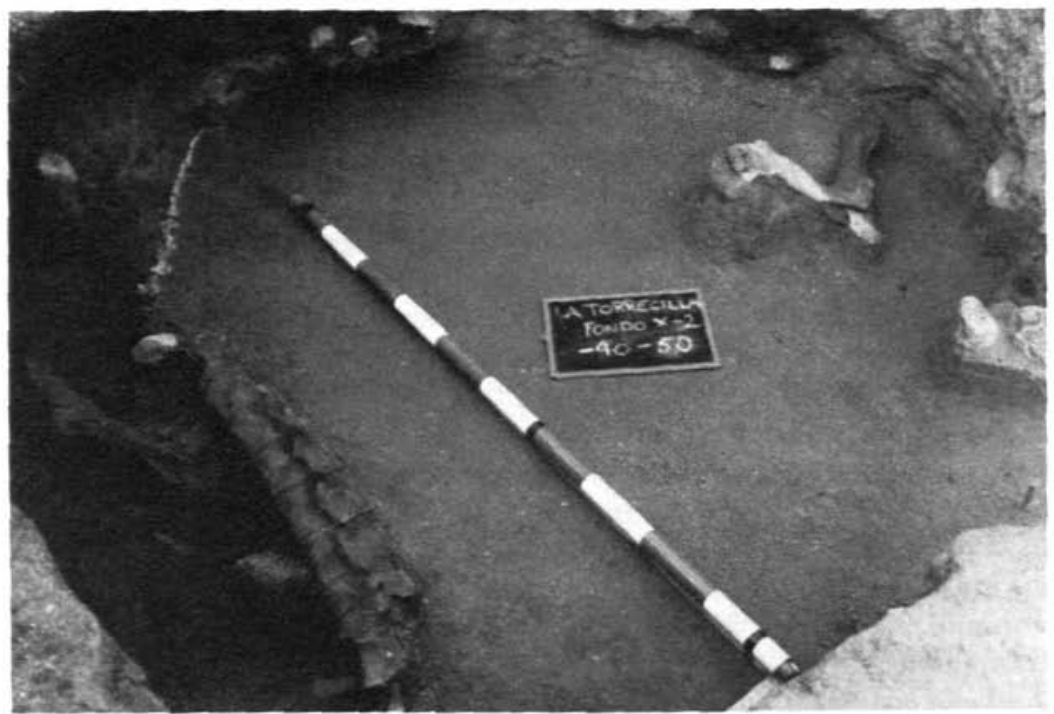

LAMINA 1-2.-Restos de bóvido colocados en conexión anatómica, en el fondo número 2. 


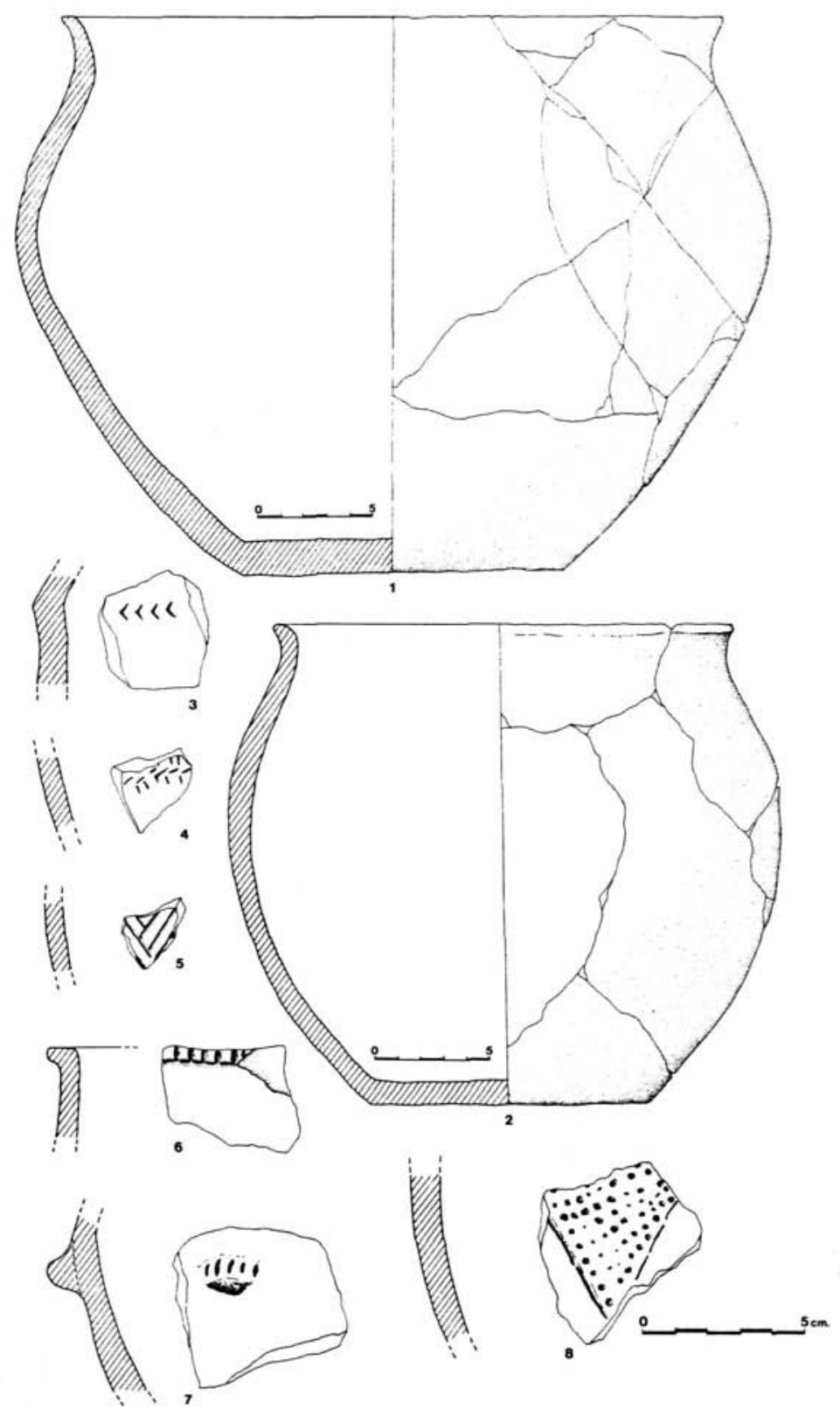

FIG. 1.-Materiales cerámicos obtenidos en el fondo número 1. Figuras 1-1 y 1-2: recipientes completos colocados boca abajo en el centro del fondo. Figuras 1-3 a 1-8 fragmentos cerámicos decorados hallados en la parte inferior del fondo y en el nivel superior de cierre. 

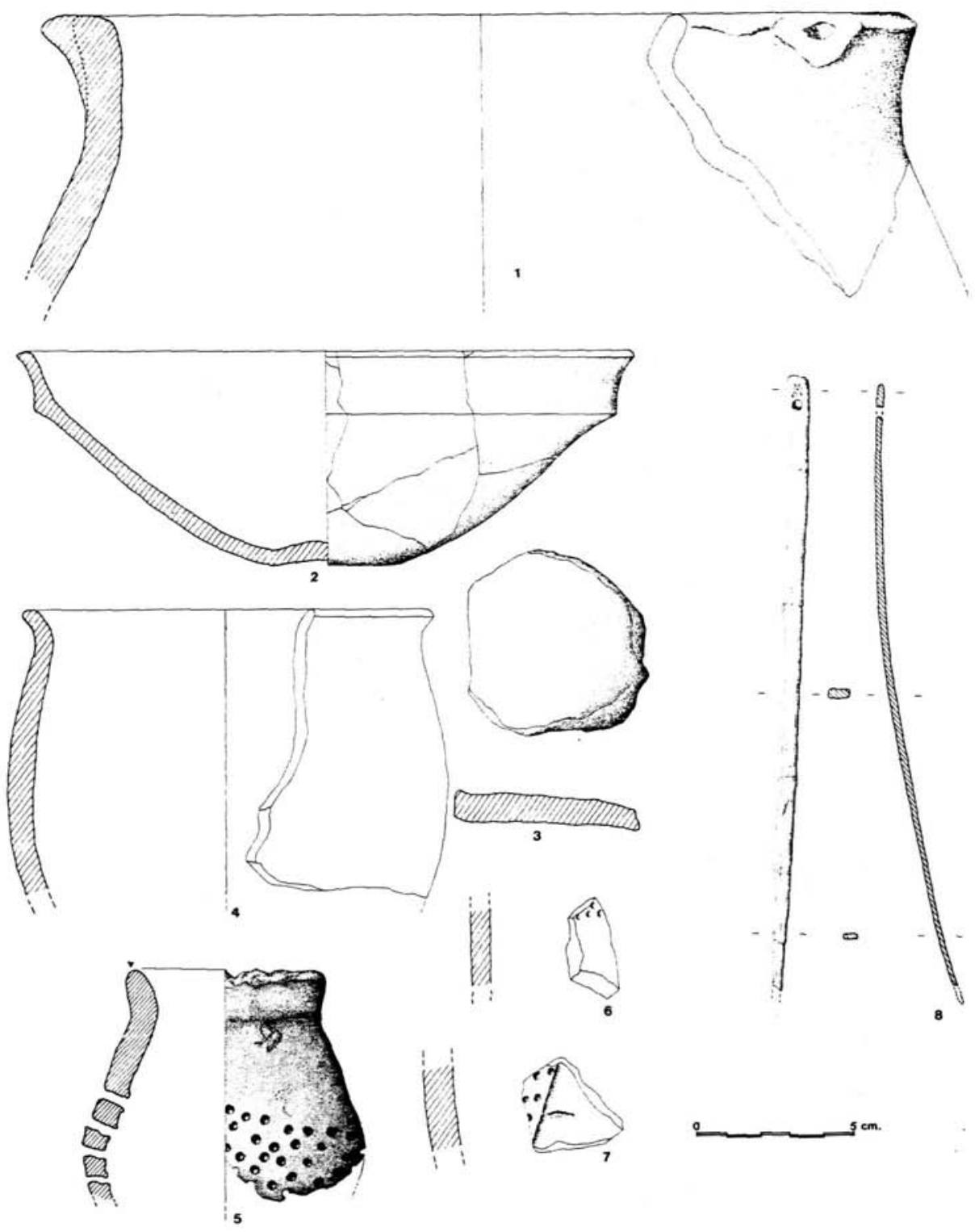

FIG. 2.-Materiales cerámicos y aguja ósea encontrados en el fondo número 2. Figuras 2-1 y 2-7: fragmentos cerámicos aparecidos en el nivel superior de cierre junto a abundantes restos óseos. Figuras 2-2; 2-3; 2-5; y 2-7: plato, recorte discoidal, encella y aguja de hueso depositados junto al bóvido colocado, en conexión anatómica en el centro del fondo. Figuras 2-4 y 2-6 fragmentos de cerámica encontrados en la base del fondo. 
ba una forma de saco con un cuello cilíndrico bien marcado y un cuerpo de tendencia hemiesférica, sensiblemente más ancho en la base. Sus dimensiones máximas eran un metro de altura por 1,40 metros de anchura.

Una vez limpia la totalidad de la bolsada se observó que en un punto de la parte inferior la tierra oscura seguía apareciendo, haciendo imposible la delimitación de las paredes en esta zona, ello nos indujo a pensar en la existencia de una segunda bolsada intersectada a la altura de la zona inferior de las paredes. En efecto, pronto se comprobó, tanto en superficie, como en el corte del arenero, la existencia de una segunda bolsada o "fondo" geminado al primero, por lo que se procedió a la excavación del segundo fondo. En él, tras la limpieza de la capa más superficial, se observó la presencia de una mancha oscura, relativamente extensa, en la que se recogieron algunos fragmentos de terra sigillata junto a restos de un posible hogar; por debajo de éste se encontraron tejas y pequeños fragmentos de pasta vítrea. Este "suelo de ocupación" romano tenía una potencia de 30 centímetros, nivel a partir del cual se delimitaba perfectamente la boca del segundo "fondo" prehistórico, de planta completamente circular y con un diámetro, en esta zona de la boca, de unos 55 centímetros.

A partir de los 30 centímetros bajo la superficie actual, desaparecerían los restos del suelo de ocupación romano y se delimitaba con claridad, la boca de este segundo fondo que presentaba una planta netamente circular, con un diámetro de 55 centímetros. La primera capa del fondo, de unos 5 centímetros de potencia, aparecía fuertemente cementada, posiblemente como consecuencia del endurecimiento producido por las altas temperaturas del "hogar» romano. Bajo esta primera capa encontramos, en un nivel similar al de la concentración de restos más superficiales del fondo 1, una serie de fragmentos cerámicos, entre ellos uno decorado con un triángulo inciso relleno de puntillado (fig. 2-7) y 24 huesos de los cuales la mayoría pertenecen a bos y el resto son de ovicápridos, cervus y sus. Estos huesos no estaban en conexión anatómica pero algunos parecían estar intencionadamente dispuestos, concretamente, los astrágalos de ovicáprido y bos se encontraron clavados a ambos lados de una tibia.

Por debajo de este conjunto los hallazgos eran mucho más esporádicos, recogiéndose sólo algunos fragmentos más, muy deshechos, y restos cerámicos de escaso tamaño. En cambio, a 65 centímetros de la superficie y a unos 30 centímetros bajo la concentración de huesos y cerámica que acabamos de describir, se descubrió un nivel, coincidiendo con la parte más ancha del fondo, en el que subyacían una extremidad delantera de un bóvido juvenil colocado en conexión anatómica y la parte posterior del tronco y extremidad inferior también en conexión anatómica, de otro bóvido, en este caso adulto. Sobre estos restos descansaba un pequeño plato de superficie bruñida y carena alta (fig. 2-2); un fragmento de colador o "encella" (fig. 2-5); un recorte discoidal de cerámica (fig. 2-3) y una aguja de hueso (fig. 2-8) de 20 centímetros de longitud a la que sólo le faltaba el extremo terminal 
(8), la cual estaba cuidadosamente colocada en paralelo a la cola del animal. En este nivel en el que se depositaron los restos animales, el perímetro del fondo estaba resaltado con una serie de pequeños cantos rodados colocados junto a la pared, a tramos regulares. Junto a ellos se observaban también pequeños carboncillos y restos de tierras quemadas de escasas proporciones.

Bajo la superficie en la que se encontraban los restos animales, la tierra que rellenaba el "fondo" tenía una coloración más clara y de tonalidad amarillenta, lo cual parecía evidenciar un menor contenido orgánico, resultando arqueológicamente estéril en unos 8 centímetros de potencia. La base del "fondo", situada a 10 centímetros por debajo de los depósitos de animales, presentaba una coloración grisácea y contenía minúsculos carboncillos; en este estrato se obtuvieron, además, varios fragmentos óseos y cerámicos, uno de éstos con decoración puntillada (fig. 2-6). La fauna representada en este nivel inferior es sus y ovicáprido.

Este halllazgo cuyos restos cerámicos y óseos permiten adscribirlo al Horizonte Cogotas I, posiblemente en un momento inicial, viene a plantearnos una vez más la funcionalidad de los "fondos" u hoyas excavadas (9) en el subsuelo confirmando que no siempre deben interpretarse como simples basureros donde eran recogidos los deshechos producidos por las sucesivas ocupaciones humanas, ni tampoco responden, en este caso, a silos.

En nuestra opinión los dos "fondos" geminados (fig. 3) parecen formar un conjunto correspondiente a un mismo momento y realizado con un mismo sentido, posiblemente votivo. Aunque la sincronía de la ejecución de los dos fondos no está plenamente confirmada con la presencia de fragmentos de una misma pieza en ambos fondos, ni con la absoluta seguridad de que algunos de los restos óseos encontrados en ambos fondos pertenezcan al mismo individuo, hay indicios suficientes para suponer esta sincronía. En primer lugar hay que destacar que ambos "fondos" parecen estar hechos por la misma mano ya que presentan no sólo una semejanza formal muy clara sino también poseen un volumen muy similar. En segundo lugar, resulta evidente que los materiales de uno y otro "fondo" guardan una relación bastante estrecha en su colocación ya que los "cuerpos" o panzas de sendas hoyas encierran conjuntos constituidos por materiales bastante completos y cuidadosamente depositados, habiendo sido sellados en la zona de la boca por un nivel formado por abundantes huesos animales y fragmentos cerámicos de piezas ya amortizadas.

La posible función votiva del conjunto la basamos en la ausencia casi total de material entre las tierras que constituyen el relleno geológico a excepción de los materiales de gran tamaño o dispuestos con cierta intencionalidad en la zona central de sendos "fondos" y de los que se concentran en la zona de la boca creando

(8) Una pieza similar se encontró en un yacimiento del Horizonte Cogotas I, muy próximo a este hallazgo:

Martínez Navarrete, M. I. y Méndez Madariaga, A.: "Arenero de Soto. Yacimiento de fondos de cabaña del Horizonte Cogotas I», en Estudios de Prehistoria y Arqueología madrileños, n. ${ }^{\circ}$ 2, Madrid, 1983, pág. 230, fig. 17-2,

(9) Martínez Navarrete, M. I.: «El yacimiento de la Esgaravita (Alcalá de Henares, Madrid), y la cuestión de los llamados fondos de cabaña del Valle del Manzanaresn, en Frabajos de Prehistoria, 36, Madrid, 1979, págs. 83-118. 


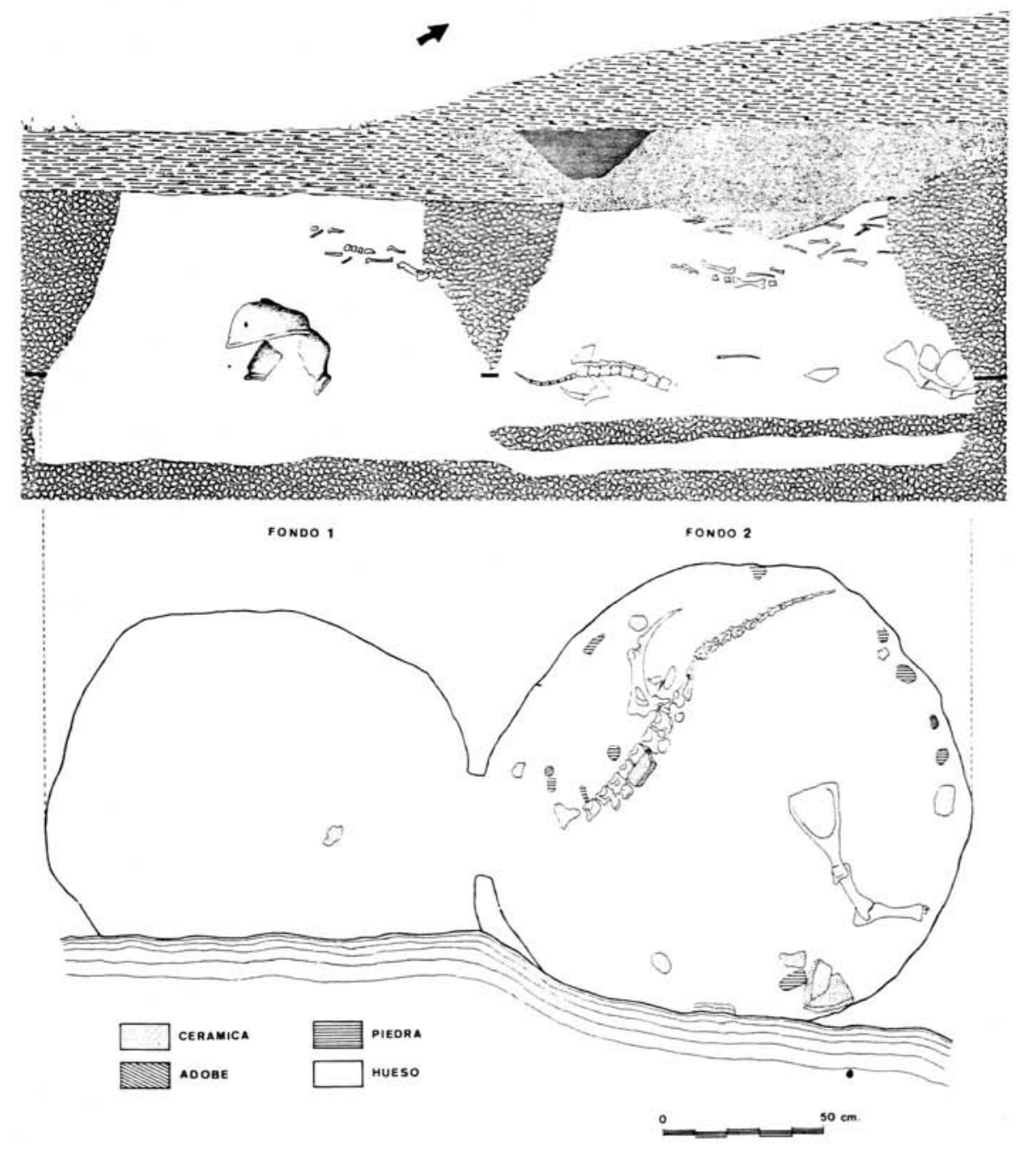

FIG. 3.-Sección y planta de los fondos 1 y 2, con la ubicación de los hallazgos más destacables.

un verdadero nivel de sellado. Este hecho contrasta fuertemente con la aparente aleatoriedad con la que generalmente se dispersa el material cerámico y óseo en otros casos.

Sin embargo, no estamos ante un hallazgo totalmente nuevo, al contrario, empiezan a ser ya muy numerosos los ejemplos que conocemos de restos óseos anima- 
les en conexión anatómica depositados en "fondos" adscribibles a diferentes horizontes culturales y también de recipientes cerámicos completos colocados hacia abajo, e incluso, tampoco es nueva la asociación de uno y otro hallazgo aunque sí lo es su relación en dos hoyas geminadas.

Los depósitos de animales completos o de partes más o menos grandes de ellos en fondos u hoyas excavados en el subsuelo es, especialmente, frecuente en yacimientos de la cuenca del Jarama-Henares pertenecientes a las facies del Bronce Clásico. El primer ejemplo conocido por la bibliografía corresponde al yacimiento del Tejar del Sastre, en uno de cuyos fondos (el núm. 44) se encontró un esqueleto completo de perro (10). En 1985, en el Sector III de Getafe, en una excavación de urgencia practicada en un yacimiento muy arrasado, obtuvimos otro esqueleto completo de un pequeño suido (posiblemente un cochinillo) de unos 6 meses de edad, asociado a unos pequeños recipientes cerámicos. En este mismo yacimiento encontramos en otro fondo, también en conexión anatómica, una extremidad inferior de un viejo bóvido, con claros indicios de haber sido utilizado para la tracción por su fuerte deformación; sobre la pata se habían colocado, boca abajo, dos grandes cazuelas (11).

Pero, sin duda los restos más espectaculares y significativos los encontramos en La Loma del Lomo (Cogolludo, Guadalajara), donde se ha localizado una necrópolis del mismo horizonte del Bronce Clásico cuyas tumbas están incluidas en "fondos" de características similares a los nuestros en las que, con frecuencia, los cadáveres tienen como única ofrenda animales completos o partes de ellos. Las especies faunísticas representadas en dichas ofrendas son: bóvido, oveja y suido (12).

Por el contrario, los ejemplos en los que encontramos recipientes colocados hacia abajo, se inscriben dentro del Horizonte Cogotas I, incluida su fase inicial. El hallazgo más próximo en el espacio y también en su cronología, lo tenemos en el propio término de La Torrecilla, a sólo un kilómetro de este conjunto, donde hace unos años se excavó un gran fondo que contenía, junto a pequeños fragmentos de cerámica decorada con características muy similares a las nuestras, ocho vasijas completas, siete de ellas hacia abajo y la octava tumbada (13). Todas ellas estaban completamente vacías, son lisas y tienen una morfología muy similar a las que presentamos, aunque son de tamaño mucho mayor.

Otros hallazgos similares se han registrado en La Muela de Alarilla (Guadalajara) (14) y en El Negralejo (Rivas-Vaciamadrid, Madrid) (15); en este último caso

(10) Quero, S.: “El poblado del Bronce Medio de El Tejar del Sastre (Madrid)", en Estudios de Prehistoria y Arqueologia Madrileños, n. ${ }^{\circ}$ 1, 1982, pág. 218.

(11) Blasco, M. C. y Barrio, J.: “Excavaciones de dos nuevos asentamientos prehistóricos en Getafe (Madrid)", en Noticiario Arqueológico Hispánico, n. ${ }^{\circ}$ 27, Madrid, 1986, págs. 75-142.

(12) Valiente Malla, J.: «Enterramiento de rito argárico en la Meseta.' El poblado de La Loma del Lomo (Cogolludo, Guadalajara)", Revista de Arqueología, año VIII, n. ${ }^{\circ} 73$, mayo 1987, págs. 34-43.

(13) Cerdeño, M. L. et alii: vid. nota n. 7, págs. 220-222.

(14) Méndez Madariaga, A. y Velasco Steigrad, F.: «La Muela de Alarilla. Un Yacimiento de la Edad del Bronce en el Valle medio del río Henares», en Revista de Arqueología, año V, n. ${ }^{\circ}$ 37, pág. 13, foto inferior.

(15) Blasco Bosqued, M. C.: "Un nuevo yacímiento del Bronce madrileño. El Negralejo, RivasVaciamadrid», en Noticiario Arqueológico Hispánico, núm. 17, Madrid, 1983, págs. 145-190. 
se encontró un recipiente colocado hacia abajo, descansado sobre la base de un "fondo" y conteniendo en su interior un gran núcleo de sílex. Estos dos yacimientos corresponden a una fase clásicas del Horizonte Cogotas I.

Si en el caso de La Loma del Lomo, no existen duda sobre la función de los restos animales como ofrendas funerarias, en el resto de los yacimientos la intencionalidad de estos depósitos no es tan evidente. De todas formas, creemos que este tipo de hallazgos debió de responder siempre a una finalidad más ritual que funcional, ya que debe descartarse la posibilidad de "almacenes de carne" pues no parece posible la conservación de la carne en estas "hoyas" donde no existe ningún tipo de aislamiento. Por otra parte, entre los depósitos hay perro que es un animal que no suele ser consumido, así como la parte final de las patas de los bóvidos que tampoco son aprovechables por no proporcionar apenas carne. En otras ocasiones, como el hallazgo que nos ocupa, aunque las partes del animal encontradas son aptas para el consumo, su colocación junto a piezas completas no amortizadas, como son el pequeño plato o la aguja de hueso o incluso la "quesera", parecen indicar una preocupación simbólica. Preocupación que también se advierte en esa reiterada costumbre de colocar recipientes hacia abajo, sin otros materiales, o sobre los propios restos animales, pues no vemos sentido a la explicación que se les ha dado, de que se trata de orzas de almacenaje reservadas para una nueva reocupación del lugar. Explicación que en todo caso, sólo sería válida para los grandes recipientes de Alarilla o de La Torreciila pero no, para nuestras pequeñas vasijas, una de las cuales contenía, además, huesos animales.

En nuestra opinión estamos ante un ritual de ofrendas animales ampliamente constatado entre los pueblos peninsulares de nuestra Pre y Protohistoria hasta la II Edad del Hierro, momento del que tenemos incluso referencias escritas sobre este tipo de ritos. Durante el Bronce Final que es la época que ahora nos ocupa, creemos que estas manifestaciones pueden responder a causas muy distintas, explicándose así la existencia de fórmulas también muy diferentes. El incremento de las ofrendas animales y, más concretamente de determinadas especies domésticas en este momento, puede deberse al auge de la ganadería y a la mayor intervención de algunos animales como bóvidos, équidos y perros en tareas pastoriles y agrícolas, como colaboradores del hombre.

\section{Inventario de fauna. Perales del Río (Getafe)}

(Arturo Morales y M. ${ }^{a}$ Luz Sánchez Capilla)

Fondo 1

* Bos taurus:

Neurocráneo (juvenil)

1P/M (premolar o molar de maxilar)

mandíbula (juvenil, aproximadamente menor de dos años)

mandíbula (adulto)

1 vértebra lumbar 
2 costillas (una de ellas en el interior de la olla)

2 metacarpos (uno de ellos de identificación dudosa)

(nota: al menos hay restos de 2 vacas: un individuo juvenil y otro adulto)

* Ovis Aries/Capra Hircus (indeterminado):

$\mathrm{M} / \mathrm{M}$ (1 molar 1 ó 2 superior)

M (1 molar 3 superior)

diáfisis de radio

1 tibia

2 falanges primeras (F-I)

en conexión anatómica: 3 vértebras lumbares

1 sacro

* Canis Familiaris:

1 fémur

Fondo 2

a) Restos en conexión anatómica en el interior del fondo.

* Bos Taurus:

- Individuo en conexión anatómica correspondiente a un adulto de unos 6 años de edad con los centros vertebrales (+), es decir soldados. El número total de restos identificados es de 24 :

4 vértebras lumbares

1 sacro en tres fragmentos

12 vértebras caudales

3 costillas

1 hemipelvis izquierda

3 fragmentos de hemipelvis sin asignación

- Extremidad delantera de un individuo juvenil aproximadamente de 3 años que se presentó en conexión anatómica. El número total de restos identificados es de 10:

1 escápula fragmentada

1 húmero (diáfisis más 3 esquirlas, 2 distales y 1 diafisaria derecha)

1 epífisis distal derecha de radio

2 ulnas (porción de diáfisis y olocrano (-) derecho)

4 carpales derechos

1 cabeza femoral derecha y trocantes mayor derecho, ambos sin fusionar.

b) Restos de fauna situados en el nivel superior del fondo (en la boca del mismo).

* Bos Taurus: El número de restos identificados es de 12: esquirlas de neurocráneo

1 mandíbula con fractura reciente

2 fragmentos de costillas 
1 diáfisis de radio

1 diáfisis proximal de metacarpo

1 diáfisis de tibia

1 astrágalo derecho

4 fragmentos de clavija ósea

* Ovicápridos (O/C): El número de restos identificados es de 9:

1 centro de vértebra cervical

1 arco neural de vértebra cervical

3 costillas

1 escápula

1 tibia (porción distal)

2 astrágalos

* Cervus Elaphus: El número de restos identificados es de 2:

1 epífisis proximal de tibia

1 porción distal de tibia

* Sus:

1 porción distal de tibia (pero es dudosa su asignación a esta especie).

c) Restos óseos identificados ajenos a los grupos arriba citados:

* Bos Taurus:

Fragmento de diente superior (Nivel $-30 /-40 \mathrm{~cm}$.).

* O/C:

3 fragmentos de costillas (Nivel $-60 /-68 \mathrm{~cm}$.).

* Sus:

1 fragmento de mandíbula con dientes (Nivel $-60 /-68 \mathrm{~cm}$.). 